\title{
PARSING HEAD-DRIVEN PHRASE STRUCTURE GRAMMAR
}

\author{
Derek Proudian and Carl Pollard \\ Hew lett-Packard Laboratories \\ 1501 Page Mill Road \\ Palo Alto, CA. 94303, USA
}

\begin{abstract}
The Head-driven Phrase Structure Grammar project (HPSG) is an English language database query system under development at Hewlett-Packard Laboratories. Unlike other product-oriented efforts in the natural language understanding field, the HPSG system was designed and implemented by linguists on the basis of recent theoretical developments. But, unlike other implementations of linguistic theories, this system is not a toy, as it deals with a variety of practical problems not covered in the theoretical liternture. We believe that this makes the HPSG system unique in its combination of linguistic theory and practical application.

The HPSG system differs from its predecessor GPSG, reported on at the $1982 \mathrm{ACL}$ meeting (Gawron et al. $\{1982 \mid)$, in four significant respects: syntax, lexical representation, parsing, and semantics. The paper focuses on parsing issues, but also gives a synopsis of the underlying syntactic formalism.
\end{abstract}

\section{Syntax}

HPSG is a lexically based theory of phrase structure, so called because of the central role played by grammatical heads and their associated complements.' Roughly speaking, heads are linguistic forms (words and phrases) that exert syntactic and semantic restrictions on the phrases, called complements, that characteristically combine with them to form larger phrases. Verbs are the heads of verb phrasess (and sentences), nouns are the heads of noun phrases, and so forth.

As in most current syntactic theories, categories are represented as complexes of feature specifications. But the HPSG treatment of lexical subcalegorization obviates the need in the theory of calegories for the notion of bar-level (in the sense of X-bar theory, prevalent in much current linguistic research). In adrition, the augmentation of the system of categories with stackvalued fealures - features whose values are scquences of categories - unifies the theory of lexical subcategorization with the theory of binding phenoment. By binding phenomena we moan essentially non-clausebounded dependencies, such as those involving dislocated constituents, relalive and interrogative pronouns, and reflexive and reciprocal pronouns $\{12\}$.

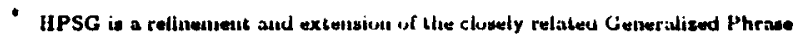
Structure Granumar [7]. The details uf the theury of IIPS(; are wet torth in [11].
More precisely, the subcategorization of a head is encoded as the value of a stack-valued feature called "SUBCAT". For example, the SUBCAT value of the verb persuade is the sequence of three categories (VP, NP, NP|, corresponding to the grammatical relations (GR's): controlled complement, direct object, and subject respectively. We are adopting a modified version of Dowty's |1982| terminology for GR's, where subject is last, direct object second-to-last, etc. For semantic reasons we call the GR following a controlled complement the controller.

One of the key differences between HPSG and its predecesor GPSG is the massive relocation of linguistic information from phrase structure rules into the lexicon $[5]$. This wholesale lexicalization of linguistic information in HPSG results in a drastic reduction in the number of phrase structure rules. Since rules no longer handle subcategorization, their sole remaining function is to encode a small number of language-specific principles for projecting from lexical entries l.u surface constituent order.

The schematic nature of the grammar rules allows the system to parse a large fragment of English with only a small number of rules (the system currently uses sixteen), since each rule can be used in many different situations. The constituents of each rule are sparsely annotated with features, but are fleshed out when taken together with constituents looked for and constituents found.

For example the sentence The manager works can be parsed using the single rule $\mathrm{Rl}$ below. The rule is applied to build the noun phrase The manager by identifying the head $H$ with the lexical element manager and the complement $C l$ with the lexical element the. The entire sentence is built by identifying the $H$ with works and the CI with the noun phrase described above. Thus the single rule RI functions as both the $S$ $\rightarrow N P V l^{\prime}$, and $N P \rightarrow$ Det $N$ rules of familiar context free gramtiars.

$$
\begin{aligned}
& \text { R1. } x \rightarrow \text { cl } h[(\text { CONTROL INTRANS })] \text { a* } \\
& \text { Figure 1. A Grammar Rule. }
\end{aligned}
$$




\section{Feature Passing}

The theory of HPSG embodies a number of substantive hypotheses about universal grammatical principles. Such principles as the Head Feature Principle, the Binding Inheritance Principle, and the Control Agreement Principle, require that certain syntactic features specified on daughters in syntactic trees are inherited by the mothers. Highly abstract phrase structure rules thus give rise to fully specified grammatical structures in a recursive process driven by syntactic information encoded on lexical heads. Thus HPSG, unlike similar "unification-based" syntactic theories, embodies a strong hypothesis about the flow of relevant information in the derivation of complex structures.

\section{Unification}

Another important difference between HPSG and other unification based syntactic theories concerns the form of the expressions which are actually unified. In HPSG, the structures which get unified are (with limited exceptions to be discussed below) not general graph structures as in Lexical Functional Grammar [1], or Functional Unification Grammar |10|, but rather flat atomic valued feature matrices, such as those shown below.

\section{[(CONTROL O INTRANS) (MAJ N A) (AGR 3RDSG) (PRD MINUS) (TOP MINUS)]}

\section{[(CONTROL O) (MAJ N V) (INV PLUS)]}

\section{Figure 2. Two feature matrices.}

In the implementation of HPSG we have been able to use this restriction on the form of feature matrices to good advantage. Since for any given version of the system the range of atomic features and feature values is fixed, we are able to represent flat feature matrices, such as the ones above, as vectors of inicgers, where each cell in the vector represents a feature, and ihe integer in each cell represents a disjunction of the possible values for that feature.

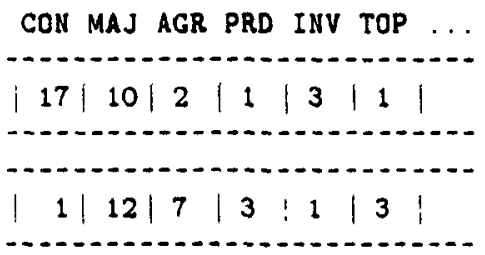

Figure 3: Two transduced feature matrices.

For example, if the possible values of the $M A J$ feature are $N, V, \Lambda$, and $P$ then we can uniquely represent any combination of these fealures with an inleger in the range $0 . .15$. This is accomplished simply by assigning each possible value an index which is an integral power of 2 in this range and then adding up the indices so derived for each disjunction of values encountered. Unification in such cases is thus reduced to the "logical and" of the integers in each cell of the vector representing the feature matrix. In this way unification of these flat structures can be done in constant time, and since "logical and" is generally a single machine instruction the overhead is very low.

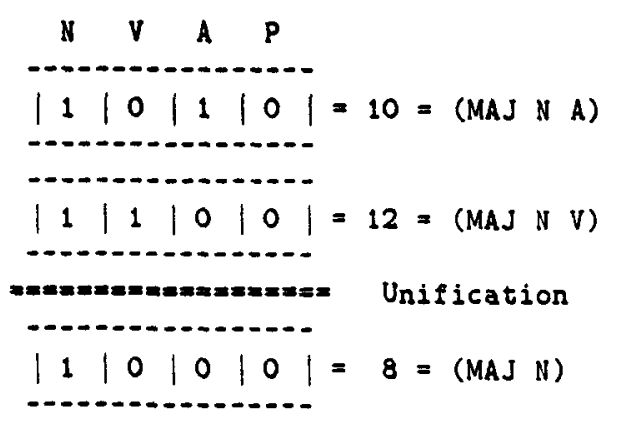

Figure 4: Closeup of the MAJ feature.

There are, however, certain cases when the values of features are not atomic, but are instead themselves feature matrices. The unification of such structures could, in theory, involve arbitrary recursion on the general unification algorithm, and it would seem that we had not progressed very far from the problem of unifying general graph structures. Happily, the features for which this property of embedding holds, constitute a small finite set (basically the so called "binding features"). Thus we are able to segregate such features from the rest, and recurse only when such a "category valued" feature is present. In practice. therefore, the time performance of the general unification algorithm is very good, essentially the same as that of the Hat structure unification algorithm described above.

\section{Parsing}

As in the earlier GPSG system, the primary job of the parser in the HPSG system is to produce a semantics for the input sentence. This is done compositionally as the phrase structure is built, and uses only locally available information. Thus every constituent which is built syntactically has a corresponding semantics built for it at the same time, using only information available in the phrasal subtree which it immediately dominates. This locality constraint in computing the semantics for constituents is an essential characteristic of HPSG. For a more complete description of the semantic treatment used in the HPSG system see Creary and Pollard $\{2 \mid$.

\section{Head-driven Active Chart Parser}

A crucial dilference between the HPSG system and its predecessor GPSG is the importance placed on the head constituent in HPSG. In HPSG it is the head constituent of a rule which carries the subcategorization information needed to build the other constituents of 
the rule. Thus parsing proceeds head first through the phrase structure of a sentence, rather than left to right through the sentence string.

The parser itself is a variation of an active chart parser $[4,9,8,13]$, modified to permit the construction of constituents head first, instead of in left-to-right order. In order to successfully parse "head first", an edge" must be augmented to include information about its span (i.e. its position in the string). This is necessary because head can appear as a middle constituent of a rule with other constituents (e.g. complements or adjuncts) on either side. Thus it is not possible to record all the requisite boundary information simply by moving a dot through the rule (as in Earley), or by keeping track of just those constituents which remain to be built (as in Winograd). An example should make this clear.

Suppose as before we are confronted with the task of parsing the sentence The manager works, and again we have available the grammar rule $R 1$. Since we are parsing in a "head first" manner we must match the $H$ constituent against some substring of the sentence. But which substring? In more conventional chart parsing algorithms which proceed left to right this is not a serious problem, since we are always guaranteed to have an anchor to the left. We simply try building the leftmost constituent of the rule starting at the leftmost position of the string, and if this succeeds we try to build the next leftmost constituent starting at one position to the right of wherever the previous constituent ended. However in our case we cannot assume any such anchoring to the left, since as the example illustrates. the $H$ is not always leftmost.

The solution we have adopted in the HPSG system is to annotate each edge with information about the span of substring which it covers. In the example below the inactive edge $E 1$ is matched against the head of rule $R 1$, and since they unify the new active edge $\mathrm{E} 2$ is crealed with its head constituent instantiated with the fealure specifications which resulted from the unitication. This new edge E2 is annotated with the span of the inactive edge $E 1$. Some time later the iractive edge E3 is matched against the "np" constitlent of our active edge E2, resulting in the new active edge E.t. The span of $E 4$ is obtained by combining the starting position of E3 (i.e. 1) with the finishing postion of E2 (i.e. $3)$. The point is that edges are constructed from the head out, so that at any given time in the life cycle of an edge the spanning information on the edge records the span of contiguous substring which it covers.

Note that in the transition from rule $R I$ to edge E2 we have relabeled the constituent markers $x, c 1$, and $h$ with the symbols $s, n p$, and $V P$ respectively. This is done merely as a mnemonic device to reflect the fact that once the hend of the edge is found, the subcategorization information on that head (i.e. the values of the "SUBCAT" feature of the verb works) is

\footnotetext{
"An edge is, loovedy opeaking, an instantiation of a rtile with snuse of the features on conotituents made more specitic
}

propagated to the other elements of the edge, thereby restricting the types of constituents with which they can be satisfied. Writing a constituent marker in upper case indicates that an inactive edge has been found to instantiate it, while a lower case (not yet found) constituent in bold face indicates that this is the next constituent which will try to be instantiated.

$$
\begin{aligned}
& \text { E1. } V\langle 3,3\rangle \\
& \text { R1. } x \rightarrow c 1 \text { b a* } \\
& \text { E2. } 8\langle 3,3\rangle \rightarrow \text { np VP a* } \\
& \text { E3. NP<1,2> } \\
& \text { E2. } s<3,3\rangle \rightarrow \text { np VP a* } \\
& \text { E4. } s<1,3\rangle \rightarrow \text { VP VP } a *^{*}
\end{aligned}
$$

Figure 5: Combining edges and rules.

\section{Using Semantics Restrictions}

Parsing "head first" offers both practical and theoretical advantages. As mentioned above, the calegories of the grammatical relations subcategorized for by a particular head are encoded as the SUBCAT value of the head. Now GR's are of two distinct types: those which are "saturated" (i.e. do not subcategorize for any thing themselves), such as subject and objects, and those which subcategorize for a subject (i.e. controlled complements). One of the language-universal grammatical principles (the Control Agreement Principle) requires that the semantic controller of a controlled complement always be the next grammatical relation (in the order specified by the value of the SUBCAT feature of the head) after the controlled complement to combine with the head. But since the HPSG parser always finds the head of a clause first, the grammatical order of its complements, as well as their semantic roles, are always specified before the complements are found. As a consequence, semantic processing of constituents can be done on the fly as the constiluents are found, rather than waiting until an edge has been completed. Thus semantic processing can be clont extremely locally (constituent-to-constituent in the edge, racher than merely node-to-node in the parse tree as in Montague semantics), and therefore a parse path rin be abandoned on semantic grounds (e.g. sortal inconsistency) in the middle of constructing an edge. In this way semantics, as well as syntax, can be used to control the parsing process.

\section{Anaphora in HPSG}

Another example of how parsing "head lirst" pays off is illustrated by the elegant technique this strategy makes possible for the binding of intrasentential anaphors. This inethod allows us to assimilate cases of bound anaphora to the same general binding mellod used in the HPSG system to handle other non-lexicallygoverned dependencies such as gaps, inlerrogative pronouns, and relative pronouns. Roughly, the unbound dependencies of each type on every constituenil are encoded as values of an appropriate stack-valued feature 
("binding feature"). In particular, unbound anaphors are kept track of by two binding features, REFL (for reflexive pronouns) and BPRO for personal pronouns available to serve as bound anaphors. According to the Binding Inheritance Principle, all categories on binding-feature stacks which do not get bound under a particular node are inherited onto that node. Just how binding is effected depends on the type of dependency.

In the case of bound anaphora, this is accomplished by merging the relevant agreement information (stored in the REFL or BPHO stack of the constituent containing the anaphor) with one of the later GR's subcategorized for by the head which governs that constituent. This has the effect of forcing the node that ultimately unifies with that GR (if any) to be the sought-after antecedent. The difference between reflexives and personal pronouns is this. The binding feature REFL is not allowed to inherit onto nodes of certain types (those with CON'TROL value IN'TRANS), thus forcing the reflexive pronoun to become locally bound. In the case of non-reflexive pronouns, the class of possible antecedents is deternined by modifying the subcategorization information on the liciul governing the pronoun so that all the subcategorized-lor GR's later in grammatical order than the pronoun are "contra-indexed" with the pronoun (and thereby prohibited from being its antecedent). Binding then takes place precisely as with reflexives, but somewhere higher in the tree.

We illustrale this distinction with bwo examples. In sentence $\mathrm{S} 1$ below told subcategorizes for three collstituents: the subject NP Pullum, the direct object Gazdar, and the obliçue object PP about himself:" Thus either Pullum or (iuzdar are possible antecedents of himself, but not Wasow.

\section{S1. Wasow was convinced that Pullum told Gazdar about himself.}

\section{S2. W'asulv persuaded Pullum to shave him.}

In sentence $\$ 2$ shave subcalegorizes for the direct object NP him and an NP subject eventually tilled by the constituent Pullum via control. Since the subject position is contra-indexed with the pronoun, Pullum is blocked from serving as the anlecedent. The pronoun is eventually bound by the NP Wasow higher up in the tree.

\section{Heuristics to Optimize Search}

The HPSC: system, based as it is upon a carefully developed linguistic theory, has broad expressive power. In practice. lowwever, thuch of this power is often not necessary. To exploit this fact the IIPSici system uses heuristics to help roluee the search space implicitly defined by the grammar. These heuristics allow the parser to prorluce an optimally ordered agenda of edges to try based on words used in the sentence, and on constituents it has found so far.

\footnotetext{
- The preponitioni is treated enowithatly as a cake marking
}

One type of heuristic involves additional syntactic information which can be attached to rules to determine their likelihood. Such a heuristic is based on the currently intended use for the rule to which it is attached, and on the edges already available in the chart. An example of this type of heuristic is sketched below.

R1. $x \rightarrow$ Cl h a*

Heuristic-1: Are the features of $c 1$ +QUE?

Figure 6: A rule with an attached heuristic.

Heuristic-1 encodes the fact that rule Rl, when used in its incarnation as the $S \rightarrow N P V P$ rule, is primarily intended to handle declarative sentences rather than questions. Thus if the answer to Heuristic-1 is "no" then this edge is given a higher ranking than if the answer is "yes". This heuristic, taken together with others, determines the rank of the edge instantiated from this rule, which in turn determines the order in which edges will be tried. The result in this case is that for a sentence such as 53 below, the system will prefer the reading for which an appropriate answer is ta character in a play by Shakespeare", over the reading which has as a felicitous answer "Richard Burton".

\section{S3. Who is Hamlet?}

It should be emphasized, however, that heuristics are not an essential part of the system, as are the feature passing principles, but rather are used only for reasons of efficiency. In theory all possible constituents permitted by the grammar will be found eventually with or without heuristics. The heuristics simply help a linguist tell the parser which readings are most likely, and which parsing strategies are usually nost fruitful, thereby allowing the parser to coustruct the most likely reading first. We believe that this clearly differentiates HPSG from "ad hoc" systems which do not make sharp the distinction between theoretical principle and heuristic guideline, and that this distinction is an importani one if the natural language understanding prograins of today are to be of any use to the natural language prograins and theories of the future.

\section{ACKOWLEDGEMENTS}

We would like to acknowledge the valuabie assitance of lhomas Wasow and Ivan Sag in the writing of this paper. We would also like to thank Martin Kay and Stuart Shicber for their helplul comuneutiary on an earlier draft. 


\section{REFERENCES}

(1) Bresnan, J. (ed). (1982) The Mental Representation of Grammatical Relations, The MIT Press, Cambridge, Mass.

(2) Creary, L. and C. Pollard (1985)

"A Computational Semantics for Natural Language", Proceedings of the 29rd Annual Meeting of the Association for Compulational Linguistics.

[ 3] Dowty, D.R. (1982)

"Grammatical Relations and Montague Grammar", In P. Jacobson and G.K. Pullum (eds.), The Nature of Syntactic Representation D. Reidel Publishing Co., Dordrecht, Holland.

( 4) Earley, J. (1970)

"An efficient context-free parsing algorithm", CACM 6:8, 1970.

[ 5] Flickinger, D., C. Pollard. T. Wasow (1985) "Structure-Sharing in Lexical Representation", Proceedings of the "9rd Annual Meeting of the Assuciation for Compututionul Linguistics.

( 6| Gawron, J. et al. (1982)

"Processing English with a (ieneralized Phrase Structure Granumar", $A C l$ Proceedings $\cong 0$.

[ T! Gazdar, G. et al. (in press) Generalized Phrase Structure Circmmar. Blackwell and Harvard University Press.

( 8) Kaplan, R. (1) $1 \%$.)

"A General Syntactic l'rocessor", In Rustin (ed.) Natural Language Processing. Algorithmics Press, N.Y.

(9) Kay, M. (1973) "The MIND Systeu", In Ruslin (ed.) Natural Language Processing. Algorithmics Press, N.Y.

[10 $\mathbf{K a y}, \mathbf{M}$. (forthcoming) "Parsing in Functional Unilication Cirammar".

11) Pollard. C. (1981)

Generalized Conlexl-Frec Cirammars, Ilead Ciramrnurs, und Natural lathguage, l'h.D. Dissertalion, Slanford.

| 12 Pollard, C: (forthroming) "A Semantic: Approach to Binding in an Monostratal "heory", To appear in Linguistics and Philosuphy.

| $1: 3 \mid$ Winograd, T. (1980) Language as a Coynitive Process. Addison-Wesley, Readiıg, Mass. 